\title{
Time and Frequency Domain Analyses Based Expert System for Impulse Fault Diagnosis in Transformers
}

\author{
P. Purkait \\ Haldia Institute of Technology \\ City Centre, Haldia \\ Midnapore 721 657, India \\ and S. Chakravorti \\ Electrical Engineering Department \\ Jadavpur University \\ Kolkata 700032 , India
}

\begin{abstract}
The presence of insulation failure in the transformer winding is detected using the voltage and current oscillograms recorded during the impulse test. Fault diagnosis in transformers has several parameters such as the severity of fault, the kind of fault and the location of the fault. Detection of major faults involving a large section of the coils have never been a big issue and several visual and computational methods have already been proposed by several researchers. The present paper describes an expert system based on re-confirmative method for the diagnosis of minor insulation failures involving small number of turns in transformers during impulse tests. The proposed expert system imitates the performance of an experienced testing personnel. To identify and locate a fault, an inference engine is developed to perform deductive reasoning based on the rules in the knowledge base and different statistical techniques. The expert system includes both the time-domain and frequency-domain analyses for fault diagnosis. The basic aim of the expert system is to provide a non-expert with the necessary information and interaction in order to make fault diagnosis in a friendly windowed environment. The rules for fault diagnosis have been so designed that these are valid for the range of power transformers used in practice up to a voltage level of $33 \mathrm{kV}$. The fault diagnosis algorithm has been tested using experimental results obtained for a 3 MVA transformer and simulation results obtained for 5 and 7 MVA transformers.
\end{abstract}

\section{INTRODUCTION}

$\mathrm{I}^{\mathrm{s}}$ MPULSE tests are performed on power transformers in order to assess their insulation integrity when subjected to surge over-voltages. Impulse voltage sequences are generated in the laboratory and applied to transformers as per standards (International Electrotechnical Commission, IEC 76) [1]. The resulting voltage and current waveforms are recorded by oscilloscopes. Standards for impulse testing of power transformers such as IEC 722, 1982 [2] mainly set a guideline to the recognition of faults during impulse tests based on the current and voltage oscillograms recorded.

The impulse test of transformers and fault detection techniques thereof date back 50 years [3-8]. The efforts of earlier researchers [3-8] revealed several methods for fault diagnosis during impulse tests by visual comparison of the current oscillograms produced when a standard lightning impulse is applied to the HV winding at the Basic Insula- tion Level (BIL) and at a calibrating level. All these methods in spite of being straightforward and sensitive, they have several shortfalls. Such visual investigations should be carried out, and their results interpreted by persons properly trained to analyze these oscillograms. An experienced person can supposedly tell the nature of the fault from the nature of the oscillograms, but in practice this often results in controversy, since there are no generally recognized, well documented evaluation criteria.

Malewski et al [9] and Vaessen et al [10] introduced the transfer function method, which consists of the comparison of the frequency domain graphs deconvoluted from the test voltage and current records obtained at the full and reduced test levels. The transfer function method eliminates the problem of fault diagnosis in time domain due to deviation in input impulse waveform.

During the last decade, studies on location of power transformer faults during impulse tests have been carried 
out [11-13]. Vajana et al [12-13] described the algorithms for transfer function based detection of impulse faults between different sections of the winding involving $10 \%$ or more of the winding. The scope for diagnosis of minor faults, both series and shunt type, involving much less percentage of the winding section, still remains open.

However, contrary to the main philosophy, practical testing has indicated many serious problems of the transfer function method [14]. The possible sources leading to error and ambiguity in the transfer function computation are critically analyzed by Pandey et al [15]. Those can be summarized as (a) noise inherent in acquired data, (b) errors due to sampling, (c) A/D errors, (d) quantization, (e) finite record length effects and different signal processing methods being adopted, like windowing, filtering etc. Keeping in mind all the above, the present paper proposes a method that does not depend either on time-domain or on frequency-domain exclusively. But it uses a re-confirmative approach by combining the concepts for minor insulation failure diagnosis in transformers during impulse tests.

In reality, however, it is manually difficult to grasp the fault conditions and to identify the fault location because sound analytic knowledge and plenty of experience are required to judge fault conditions precisely. The kind of knowledge base required for carrying out these tasks may not be available in all the testing laboratories, particularly in developing countries. Unless the heuristics of fault diagnosis are well documented in a rule-base and the diagnosis algorithm is made transparent to the end-user, the controversies in the judgment procedure due to human factors will continue to persist. In this context, application of a reliable expert system in transformer fault diagnosis provides a good alternative approach.

In electric power systems the use of expert systems for fault diagnosis has already been presented by several researchers [16-19]. Expert Systems have also been employed for dissolved gas analysis in power transformers [20-21]. According to them, the effectiveness of such an expert system in emulating human heuristics depends on the precision and completeness of human knowledge accumulated over the years.

In this paper the proposed expert system tool aims at assisting the testing personnel to obtain unambiguous, reliable and quick decision in impulse-failure diagnosis of power transformers using both time and frequency domain analyses. To make the engineers free from the strenuous job of manual fault-diagnosis, a set of heuristic rules are compiled through consultation with the standards, literatures and the experts to ensure that the expert system detects a fault with high confidence, reliability and repeatability. These rules are coded into computer programs and are stored in the knowledge base of the expert system. The inference engine combines the heuristic rules of the rule-base along with mathematical and statistical methods of the expert system to act on the acquired data stored in the data base.

The expert system, which is implemented on a personal computer, has several fault diagnosis parameters to identify the faults occurring in the range of power transformers which are common in use for voltage ratings up to 33 $\mathrm{kV}$. The transfer functions have been determined by MATLAB software. The performance of the expert system have been tested by experimental results obtained from the analog model of a $3 \mathrm{MVA}, 33 / 11 \mathrm{kV}$ transformer and also by results obtained through the Electro-Magnetic Transient Program (EMTP) simulation for a 5 MVA, $33 / 11 \mathrm{kV}$ and also a 7 MVA, $20 / 6 \mathrm{kV}$ transformer. It is found from the test results that the expert system is capable of identifying and locating a variety of winding faults in an efficient manner with high reliability. The expert system can be used for both testing and educational purposes,

\section{THE EXPERT SYSTEM}

The structure of the designed expert system, time and frequency domain analysis based expert system for diagnosis of impulse faults in transformers (TAFEDIFT), has the following functional modules: (a) user interface, (b) knowledge base, consisting of the data base and the rule base for both time and frequency domain analyses, (c) inference engine for the time and frequency domain analysis modules, (d) explanation module and (e) updating module.

The different functional modules of the expert system are interrelated with each other in the manner as described in the Figure 1.

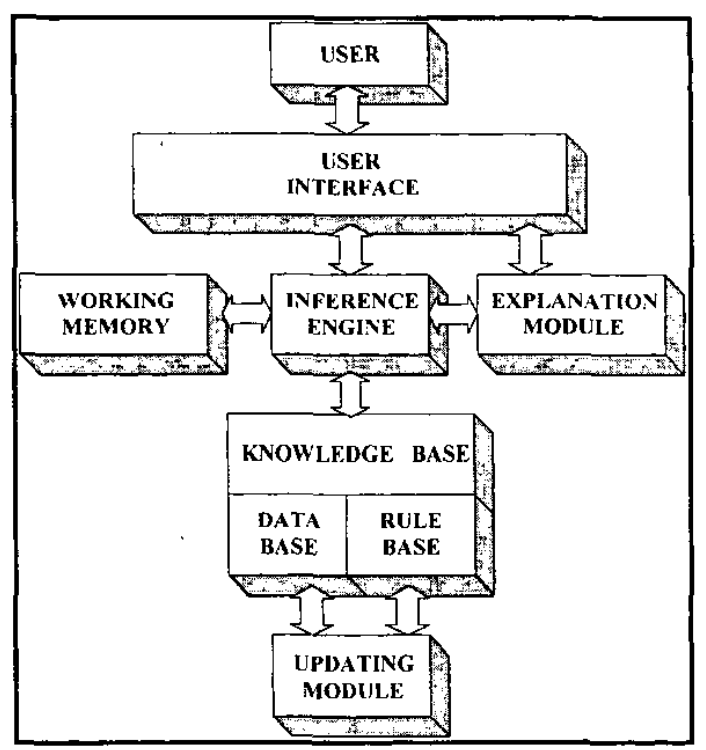

Figure 1. The Expert system configuration. 


\subsection{USER INTERFACE}

The interactive user interface (UI) using simple menus as shown in Figure 2, has been developed. With this UI, the user can ask the expert system to identify and locate the fault by using both the time domain and frequency domain analyses. The interface can display both the time-magnitude and frequency-magnitude plot and also the tabular display of the waveforms. The UI can flash the results and decision tree of the fault diagnosis. The UI is also used to update the data and rules in the knowledge base.

\subsection{KNOWLEDGE BASE}

The specialized knowledge required for the development of the expert system has been acquired from

1. Engineers working at the HV Laboratory, Jadavpur University, Kolkata, India.

2. Data accumulated from testing of different types of transformers.

3. IEEE, IEC and Indian Standards along with the information collected through various other literature surveys.

The developed knowledge-base consists of a data-base and the rule-bases for time-domain and frequency domain analysis respectively.

\subsubsection{DATA BASE}

The necessary data required for the data-base in the present work are voltage and current records (experimental or simulated as the case may be) at reduced voltage level and at full voltage level (BIL), respectively. For implementing the developed expert system the data have been collected through extensive experimentation on the analog model of a 3 MVA transformer, and the data-base have been updated by direct acquisition of the experimental results by a personal computer $(\mathrm{PC})$ via a digital storage oscilloscope. Similarly, results for variety of faults have been obtained through EMTP based models of 5 and 7 MVA transformers. The data-base has also been updated by importing these simulation results. The expert system stores and retrieves data from the data-base before the actual fault detection analysis begins.

\subsubsection{RULE BASE}

The rule-base for the time and frequency domains has been developed from the knowledge base as described above. The heuristic expert knowledge is encoded in sets of production rules with "If-then" structure in an integrated tree-like form involving both frequency and time domain analyses, as shown in the flow chart of Figure 3.

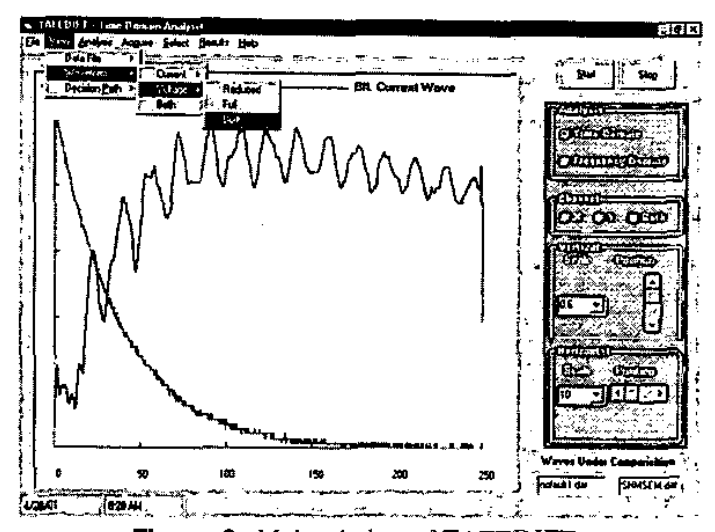

Figure 2. Main window of TAFEDIFT.

\subsection{CLASSIFICATION OF FAULTS}

Insulation failures may result in three kinds of winding faults in a transformer during impulse tests, namely series, shunt and a combination of both. Series fault implies insulation failure between the discs, while shunt fault implies insulation failure between the winding and earthed components like tank, core, etc. In practice it may also happen that a series fault occurs simultaneously with a shunt fault. The diagnostics for all these three type of faults have been incorporated in the rule base of the expert system for both time and frequency domain analyses as discussed below. The acronyms used for different types of faults considered in this study are given in Table 1.

\subsection{TIME DOMAIN ANALYSIS}

The rule-base corresponding to time-domain analysis, as discussed here, consists of heuristics of fault diagnosis by comparison between two current or two voltage waveforms, as the case may be, at reduced (calibrating wave) and full voltage level (BIL), respectively.

The different kinds of faults and their locations along the winding length are determined by several parameters. These parameters include voltage wave tail time, presence and nature of high frequency oscillations near the front of the current wave, number of oscillation peaks, peak-topeak oscillation magnitudes, times of occurrence of the respective peaks in the current wave, area under the current wave and several combinations of these parameters. The parameters have been identified through literature surveys and also in consultation with engineers in the HV Laboratory of Jadavpur University having experience of more than 30 years in the field of impulse testing of transformers.

It is well known that the lightning impulse faults in transformer windings could be detected by recording the current waveforms over a time-span of $100 \mu \mathrm{s}$. But it has been recommended $[4,8]$ that the current waveforms should be recorded over a time-span of more than $150 \mu \mathrm{s}$ for unambiguous detection, identification and location of different types of faults. Hence, in the expert system pre- 


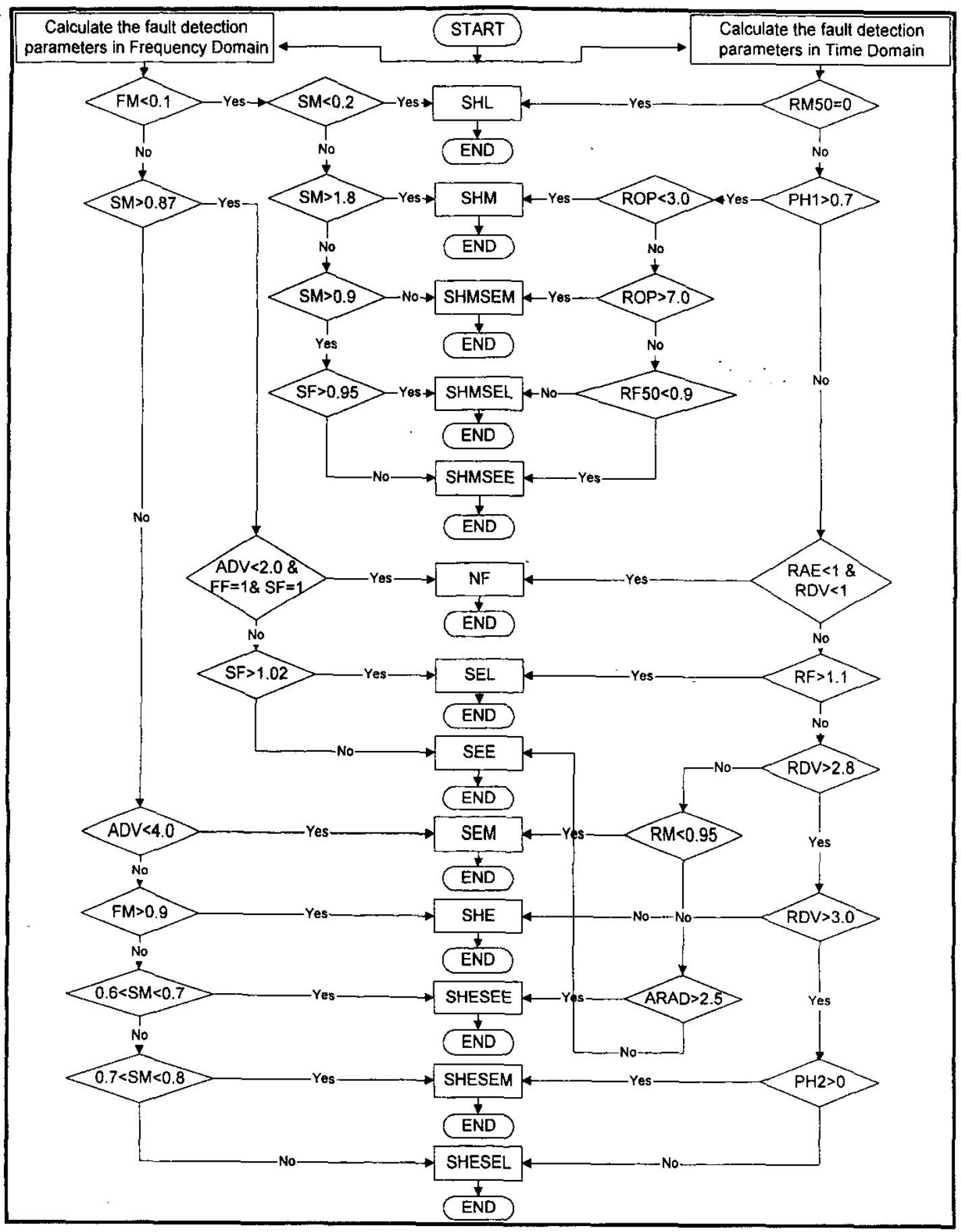

Figure 3. Knowledge tree for integrated time and frequency-domain analyses.

sented in this paper, time-domain analysis has been done over a span of $250 \mu$ s for current waveforms, so that all kinds of faults can be thoroughly diagnosed.

\subsubsection{SERIES FAULTS}

Series faults represent short circuit faults across turns or coils involving $1 \%$ of the total winding length at a time. It is observed that series fault at line end section is depicted by high frequency. oscillation of around 200-400 $\mathrm{kHz}$ within the first $50 \mu \mathrm{s}$ of the current wave. The magnitudes of oscillations in the BIL current wave are more than that in the calibrating current wave. Also the times 
of occurrence of the oscillation peaks in the BIL current wave lag their corresponding calibrating wave counterparts after $50 \mu \mathrm{s}$. Series fault at mid-winding section, however, is indicated by a deviation in the two current waveforms after a time of $50 \mu \mathrm{s}$. In such a case, although the respective times of occurrence of oscillation peaks are close between the BIL and calibrating current waves, the average peak-to-peak oscillation magnitude of the BIL current wave is less than that of the calibrating current wave. In the case of series fault at ground-end section, the oscillation peaks of the BIL current wave leads the corresponding peaks of the calibrating wave by a large amount at the later part of the wave.

\subsubsection{SHUNT FAULTS}

Shunt fault at line end section is depicted by a large initial rush of current associated with a sudden dip of the applied voltage wave. This is caused by the "reflected wave phenomenon". It is observed that this dip in voltage wave can bring down the tail time of the voltage waveform 50 $\mu s$ to as low as 25 to $40 \mu$ s along with a huge increase in the area under the curve. On the other hand, shunt fault at the mid-winding section is characterized by a remarkable increase in the number of oscillation peaks and reduction in the average peak to peak oscillation magnitude in the current wave corresponding to BIL as compared to the calibrating current wave.

In the case of shunt fault at ground-end section, the BIL current wave is similar to that due to SEL, except that the frequency of oscillation before $50 \mu \mathrm{s}$ in the BIL current wave is less than the calibrating wave and $150 \mu \mathrm{s}$ onwards, the BIL current wave is found to lead the calibrating current wave by a good amount.

\subsubsection{SIMULTANEOUS FAULTS}

The detection of simultaneous occurrence of faults within the winding needs a critical examination of the current waves. More often, more than one parameters are indicative of the nature and location of the simultaneous faults.

SHLSE: In the case of simultaneous occurrence of shunt fault at line end section along with any series fault along the length of the winding, the resulting current waveform is difficult to differentiate from that due to shunt fault alone at line end section. This is due to the fact that a shunt fault at the line end section diverts majority of the fault current to earth from the fault point and a very small amount of current actually continue to pass through the rest of the winding. As a result, the occurrence of any kind of series fault thereafter barely alters the fault current. This situation is depicted in Table 2 and Table 3 where very little deviation is observed between the fault detection parameters corresponding to SHLSE and SHL.
Table 1. List of acronyms used.

\begin{tabular}{ll}
\hline NF & No-Fault \\
SEL & Series Fault at Line-End \\
SEE & Series Fault at Mid-Winding \\
SHL & Series Fault at Earth-End \\
SHM & Shunt Fault at Line-End \\
SHE & Shunt Fault at Mid-Winding \\
SHLSE & Shunt Fault at Earth-end \\
& Simultaneous occurrence of Shunt \\
& Fault at Line-End and Series \\
fault at any other part of the winding \\
SHMSEL & Simultaneous occurrence of Shunt \\
& Fault at Mid-Winding and Series \\
& Fault at Line-End \\
SHMSEM & Simultaneous occurrence of Shunt \\
& and Series Faults at Mid-Winding \\
SHMSEE & Simultaneous occurrence of Shunt \\
& Fault at Mid-Winding and Series \\
& Fault at Earth-End \\
SHESEL & Simultancous occurrence of Shunt \\
& Fault at Earth-End and Series \\
& Fault at Line-End \\
SHESEM & Simultaneous occurrence of Shunt \\
& Fault at Earth-End and Series \\
& Fault at Mid-Winding \\
SHESEE & Simultaneous occurrence of Shunt \\
& and Series Faults at Earth-End \\
\hline
\end{tabular}

SHMSEL: The characteristic waveform of simultaneous shunt fault at mid-winding and series fault at line end section has the combined effect of both the individual faults. In such a case, the number of oscillations in the current wave at BIL is much more after a period of $50 \mu \mathrm{s}$. Also there are good amounts of high frequency $(100 \mathrm{kHz}$ or more) oscillations within the first $50 \mu$ s of the faulty current wave.

SHMSEM: Simultaneous shunt and series faults at the mid-winding section result in a small increase in the area under the envelope of the under-shoots of the current wave at BIL. The number of oscillation peaks in the BIL current wave is much more than that in the calibrating current wave. The oscillation peaks in the current wave at BIL leads the respective peaks in the calibrating current wave and the leading time difference increases as the time progresses.

SHMSEE: When a simultaneous series fault occurs at the ground-end section instead of mid-winding section, along with the shunt fault at the mid-winding section, the number of oscillations of the current wave at BIL is more than that for the calibrating current wave. Both the times of occurrence of the oscillation peaks and the areas under the curve for the BIL and calibrating current waves deviate substantially and their deviation increases with time.

SHESEL: The simultaneous occurrence of shunt fault at earth end section and series fault at line end section is recognized by a lead in the times of occurrence of the oscillation peaks of the current wave at BIL with respect to the corresponding calibrating current wave. However, the numbers of oscillation peaks are nearly the same in both the current waves. 
SHESEM: If the area under the current wave at BIL is less than the calibrating current wave and the BIL current wave leads the calibrating wave after $150 \mu$ s by a large amount, then it indicates simultaneous occurrence of shunt fault at earth end and series fault at mid-winding section.

SHESEE: In the case when the series fault is nearer to the ground end section along with a shunt fault at the ground-end, the average peak-to-peak oscillation magnitude of the current wave at BIL before $50 \mu$ s becomes close to the corresponding average peak-to-peak oscillation magnitude of the calibrating current wave, with the number of oscillation peaks being nearly same in both the current waves. The algorithm of fault-detection, identification and its localization using time-domain analysis, is presented in the integrated flow-chart shown in Figure 3.

The different variables used in the flow-chart are discussed in Appendix $\mathbf{I}$.

\subsection{FREQUENCY DOMAIN ANALYSIS}

For the frequency domain analysis, the Fast Fourier Transform (FFT) of the current and input voltage records are computed. The Transfer Function (TF) is then calculated as:

$$
\mathrm{TF}=\frac{\mathrm{FFT}(\mathrm{i})}{\mathrm{FFT}(\mathrm{v})}
$$

Table 2. Calculated values of fault detection variables in time-domain analysis.

\begin{tabular}{|c|c|c|c|c|c|c|c|c|c|c|c|c|}
\hline \multirow[t]{2}{*}{ Fault } & \multirow[t]{2}{*}{ MVA } & \multicolumn{11}{|c|}{ Time Domain Parameters } \\
\hline & & ARAD & PH1 & $\mathrm{PH} 2$ & PH3 & $\mathrm{RAE}$ & RDV & RF & RF50 & $\mathrm{RM}$ & RM50 & ROP \\
\hline \multirow{3}{*}{$\mathrm{NF}$} & 3 & 1.297 & 0.018 & -0.542 & -0.195 & 0.940 & 0.617 & 1.000 & 1.000 & 1.005 & 0.177 & 1.056 \\
\hline & 5 & 0.229 & -0.005 & 0.004 & -0.070 & 0.445 & 0.553 & 1.000 & 1.000 & 1.000 & 0.090 & 1.178 \\
\hline & 7 & 1.798 & 0.298 & 1.030 & 0.348 & 0.768 & 0.981 & 1.000 & 1.000 & 1.048 & 0.208 & 1.441 \\
\hline \multirow{3}{*}{ SEL } & 3 & 0.729 & -0.015 & -0.213 & -0.973 & 3.750 & 3.347 & 1.275 & 1.203 & 1.100 & 0.921 & 1.241 \\
\hline & 5 & 1.254 & 0.315 & -0.169 & -1.123 & 1.048 & 0.842 & 1.550 & 1.330 & 1.824 & 0.786 & 1.176 \\
\hline & 7 & 1.656 & 0.317 & -0.953 & -0.778 & 1.824 & 1.273 & 1.161 & 1.197 & 1.055 & 0.818 & 0.999 \\
\hline \multirow{3}{*}{ SEM } & 3 & 6.430 & 0.366 & -0.298 & -0.203 & 3.261 & 1.787 & 1.019 & 1.808 & 0.800 & 0.112 & 1.078 \\
\hline & 5 & 0.980 & -0 & 0.241 & -0.147 & 2.995 & 1.721 & 1.051 & 1.305 & 0.941 & 0.088 & 1.098 \\
\hline & 7 & 2.461 & 0.423 & -0.637 & -0.203 & 3.775 & 1.612 & 1.070 & 1.091 & 0.909 & 0.175 & 1.045 \\
\hline \multirow{3}{*}{ SEE } & 3 & 1.437 & -0.095 & 0.615 & -0.423 & 4.680 & 2.632 & 0.829 & 0.984 & 1.143 & 0.261 & 1.795 \\
\hline & 5 & 0.805 & -0.023 & 0.210 & -1.044 & 1.898 & 2.612 & 1.034 & 1.127 & 1.882 & 0.078 & 1.863 \\
\hline & 7 & 0.284 & -0.009 & 0.330 & 0.825 & 0.986 & 1.721 & 1.043 & 1.243 & 1.045 & 0.196 & 1.423 \\
\hline \multirow{3}{*}{ SHL } & 3 & 45.12 & 1.702 & -7.286 & -9.264 & 0.000 & 0.000 & 0.842 & 1.427 & 1.500 & 0,000 & 0.000 \\
\hline & 5 & 35.81 & - & 0.000 & 0.000 & 2.896 & 7.906 & 11.56 & 0.000 & 1.529 & 0.000 & 1.000 \\
\hline & 7 & 32.50 & - & -0.256 & -0.256 & 0.000 & 0.000 & 6.406 & 0.000 & 1.182 & 0.000 & 1.000 \\
\hline \multirow{3}{*}{ SHM } & 3 & $\overline{0.494}$ & 1.273 & 0.368 & -1.246 & 3.775 & 2.307 & 0.837 & 0.760 & 1.537 & 0.167 & 2.939 \\
\hline & 5 & 1.257 & 0.919 & -0.392 & -1.975 & 1.478 & 1.063 & 0.661 & 0.946 & 1.824 & 0.026 & 2.941 \\
\hline & 7 & 1.755 & 0.914 & -1.113 & -0.699 & 1.176 & 1.166 & 0.682 & 0.881 & 1.487 & 0.071 & 2.823. \\
\hline \multirow{3}{*}{ SHE } & 3 & 5.675 & 0.556 & 0.133 & -1.224 & 4.482 & 2.870 & 0.829 & 0.987 & 0.900 & 0.230 & 1.010 \\
\hline & 5 & 1.617 & 0.294 & 0.011 & -0.913 & 4.094 & 2.936 & 0.881 & 1.011 & 0.882 & 0.167 & 1.126 \\
\hline & 7 & 2.200 & 0.451 & 0.725 & -0.473 & 3.935 & 2.976 & 0.739 & 1.077 & 0.909 & 0.182 & 0.954 \\
\hline \multirow{3}{*}{ SHLSE } & 3 & 44.04 & 1.557 & -1.286 & -8.664 & 0.000 & 0.000 & 0.842 & 1.631 & 1.860 & 0.000 & 0.000 \\
\hline & 5 & 36.93 & - & 0.000 & 0.000 & 2.936 & 4.172 & 8.763 & 0.000 & 1.229 & 0.000 & 1.000 \\
\hline & 7 & 32.80 & - & -0.256 & -0.256 & 0.000 & 0.000 & 6.406 & 0.000 & 1.438 & 0.000 & 1.000 \\
\hline \multirow{3}{*}{ SHMSEL } & 3 & 3.356 & 0.830 & -0.270 & -1.468 & 1.649 & 1.767 & 1.017 & 1.124 & 0.900 & 0.066 & 6.793 \\
\hline & 5 & 3.979 & 0.922 & -1.314 & -0.618 & 2.172 & 1.384 & 0.897 & 1.111 & 1.059 & 0.000 & 6.482 \\
\hline & 7 & 2.141 & 0.738 & -1.041 & -0.329 & 1.618 & 1.246 & 0.720 & 1.099 & 1.045 & 0.062 & 6.384 \\
\hline \multirow{3}{*}{ SHMSEM } & 3 & 1.686 & 0.879 & -0.068 & -1.012 & 1.475 & 1.544 & 1.068 & 0.954 & 0.950 & 0.117 & 8.555 \\
\hline & 5 & 3.979 & 0.922 & -0.314 & -1.223 & 2.742 & 1.384 & 0.523 & 1.953 & 0.588 & 0.000 & 7.482 \\
\hline & 7 & 1.272 & 0.995 & -0.712 & -1.110 & 1.421 & 1.219 & 0.805 & 1.091 & 0.455 & 0.019 & 9.030 \\
\hline \multirow{3}{*}{ SHMSEE } & 3 & 2.607 & 0.875 & -0.180 & -1.622 & 1.722 & 1.618 & 0.988 & 0.783 & 0.850 & 0.038 & 6.258 \\
\hline & 5 & 1.220 & 0.918 & -0.228 & -1.176 & 1.555 & 1.107 & 0.680 & 0.743 & 0.706 & 0.021 & 3.935 \\
\hline & 7 & 1.755 & 0.914 & -0.113 & -0.699 & 1.755 & 1.047 & 0.682 & 0.881 & 0.688 & 0.071 & 6.823 \\
\hline \multirow{3}{*}{ SHESEL } & 3 & 6.184 & 0.276 & -0.654 & -1.010 & 3.666 & 3.196 & 0.962 & 0.890 & 0.900 & 0.667 & 1.048 \\
\hline & 5 & 1.483 & 0.301 & -0.047 & -0.164 & 1.097 & 4.911 & 0.873 & 1.230 & 0.824 & 0.071 & 0.997 \\
\hline & 7 & 4.214 & 0.571 & -1.029 & -1.401 & 1.018 & 3.482 & 0.596 & 0.866 & 0.864 & 0.140 & 1.066 \\
\hline \multirow{3}{*}{ SHESEM } & 3 & 0.752 & 0.115 & 0.065 & -0.763 & 3.224 & 3.075 & 0.878 & 0.946 & 0.900 & 0.568 & 1.053 \\
\hline & 5 & 2.122 & 0.277 & 0.240 & -0.853 & 1.149 & 5.006 & 0.911 & 1.223 & 0.882 & 0.058 & 1.179 \\
\hline & 7 & 4.682 & 0.461 & 0.889 & -1.379 & 2.612 & 3.126 & 0.664 & 0.791 & 0.909 & 0.112 & 1.008 \\
\hline \multirow{3}{*}{ SHESEE } & 3 & 4.032 & -0.383 & 0.382 & -0.985 & 3.063 & 1.962 & 0.960 & 0.863 & 0.950 & 0.462 & 1.133 \\
\hline & 5 & 2.929 & 0.282 & 0.169 & -0.061 & 1.133 & 0.983 & 0.898 & 1.215 & 0.987 & 0.061 & 0.983 \\
\hline & 7 & 4.017 & 0.504 & 0.733 & -1.117 & 1.218 & 0.995 & 0.789 & 0.891 & 1.091 & 0.165 & 1.025 \\
\hline
\end{tabular}


Figure 4 shows typical current and voltage records of the $33 \mathrm{kV}$ winding of a $3 \mathrm{MVA}, 33 / 11 \mathrm{kV}$ transformer, when a $1.2 / 50 \mu \mathrm{s}$ lightning impulse of positive polarity is applied. Figure 5 shows the frequency spectra of the current and voltage records obtained using FFT. The FFT analysis has been done using the MATLAB Toolbox. The transfer function of the winding involving the current and voltage wave FFT-s is shown in Figure 6 . As described by Vajana et al [13], the FFT curves for both the voltage and current waves need to be calculated up to $200 \mathrm{kHz}$ with at least 1000 sampling points. However, the first few dominant poles of the transfer function being confined within a span of $100 \mathrm{kHz}$, it is sufficient to carry out fault diagnosis based on transfer functions calculated up to a frequency of $100 \mathrm{kHz}$.
The Transfer functions (TF) were calculated for both the calibrating impulse wave as well as the impulse wave at BIL. The detection of the type of fault and its location determination is based upon several parameters such as deviation in the resonant peaks, their magnitudes as well as frequencies and the area under the curve between the two transfer functions at calibrating level and BIL, respectively. The algorithm of fault diagnosis based on frequency domain analysis is discussed below.

\subsubsection{SERIES FAULTS}

For series fault at the line end section, all the resonant pole frequencies in the BIL TF curve are more than their calibrating TF counterparts. The total area under the BIL TF curve also deviates from the area under the calibrating

Table 3. Calculated values of fault detection variables in frequency-domain analysis.

\begin{tabular}{|c|c|c|c|c|c|c|c|}
\hline \multirow[t]{2}{*}{ FAULT } & \multirow[t]{2}{*}{ MVA } & \multicolumn{6}{|c|}{ FREQUENCY DOMAIN PARAMETERS } \\
\hline & & $\widehat{\mathrm{ARA}}$ & $\mathrm{ADV}$ & $\mathrm{FF}$ & FM & SF & SM \\
\hline \multirow{3}{*}{ NF } & 3 & 1.005 & $0 . \overline{482}$ & 1.000 & $\overline{0.923}$ & $1.000^{\circ}$ & 0.979 \\
\hline & 5 & 1.018 & 1.827 & 1.000 & 0.989 & 1.000 & 1.179 \\
\hline & 7 & 1.001 & 0.080 & 1.000 & 0.999 & 1.000 & 0.996 \\
\hline \multirow{3}{*}{ SEL } & 3 & 1.080 & 8.023 & 1.000 & $0.8 \overline{46}$ & 1.038 & 1.197 \\
\hline & 5 & 0.955 & 4.475 & 1.100 & 1.086 & 1.032 & 0.877 \\
\hline & 7 & 1.035 & 3.514 & 1.053 & 0.833 & 1.035 & 1.033 \\
\hline \multirow{3}{*}{ SEM } & 3 & 0.977 & 2.324 & $0 . \overline{933}$ & 0.814 & 1.038 & 0.806 \\
\hline & 5 & 0.977 & 2.283 & 0.917 & 0.952 & 1.000 & 0.854 \\
\hline & 7 & 0.963 & 3.674 & 0.868 & 0.864 & 1.018 & 0.746 \\
\hline \multirow{3}{*}{ SEE } & 3 & 1.068 & 6.801 & 1.000 & 0.803 & 1.000 & 1.063 \\
\hline & 5 & 0.973 & 2.740 & 1.050 & 1.136 & 1.000 & 1.012 \\
\hline & 7 & 0.993 & 0.718 & 1.026 & 0.992 & 1.018 & 0.956 \\
\hline \multirow{3}{*}{ SHL } & 3 & 0.203 & 79.718 & 0.000 & 0.000 & 0.923 & 0.121 \\
\hline & 5 & 1.876 & 87.580 & 0.000 & 0.000 & 0.000 & 0.000 \\
\hline & 7 & 1.307 & 30.737 & 0.000 & 0.000 & 0.000 & 0.000 \\
\hline \multirow{3}{*}{ SHM } & 3 & 0.891 & 10.897 & 0.000 & 0.000 & 0.808 & 2.057 \\
\hline & 5 & 0.848 & 15.160 & 0.000 & 0.000 & 0.903 & 1.816 \\
\hline & 7 & 0.870 & 13.007 & 0.937 & 0.083 & 0.912 & 2.127 \\
\hline \multirow{3}{*}{ SHE } & 3 & 0.865 & 13.521 & 1.133 & 0.993 & 1.154 & 0.643 \\
\hline & 5 & 0.945 & 5.480 & 1.150 & 0.923 & 1.032 & 0.714 \\
\hline & 7 & 0.956 & 4.390 & 0.842 & 0.916 & 1.000 & 0.828 \\
\hline \multirow{3}{*}{ SHLSE } & 3 & 0.166 & 83.437 & 0.000 & 0.000 & 0.000 & 0.000 \\
\hline & 5 & 1.671 & 78.032 & 0.000 & 0.000 & 0.000 & 0.000 \\
\hline & 7 & 1.259 & 25.934 & 0.000 & 0.000 & 0.000 & 0.000 \\
\hline \multirow{3}{*}{ SHMSEL } & 3 & 0.749 & 25.103 & 0.000 & 0.000 & $0 . \overline{962}$ & 1.067 \\
\hline & 5 & 0.819 & 18.082 & 0.000 & 0.000 & 1.000 & 1.125 \\
\hline & 7 & 0.824 & 17.638 & 0.789 & 0.071 & 0.965 & 1.285 \\
\hline \multirow{3}{*}{ SHMSEM } & 3 & 0.773 & 22.676 & 0.000 & 0.000 & $\overline{1.000}$ & 0.866 \\
\hline & 5 & 0.825 & 17.534 & 0.000 & 0.000 & 1.032 & 0.868 \\
\hline & 7 & 0.753 & 24.661 & 0.000 & 0.068 & 1.088 & 0.596 \\
\hline \multirow{3}{*}{ SHMSEE } & 3 & 0.784 & 21.608 & 0.000 & 0.000 & $\overline{0.923}$ & 0.924 \\
\hline & 5 & 0.843 & 15.708 & 0.000 & 0.000 & 0.903 & 1.653 \\
\hline & 7 & 0.838 & 16.201 & 0.789 & 0.079 & 0.912 & 1.355 \\
\hline \multirow{3}{*}{ SHESEL } & 3 & 0.936 & 6.371 & 1.200 & 0.787 & 1.231 & 0.541 \\
\hline & 5 & 0.953 & 4.658 & 1.100 & 0.839 & 1.032 & 0.535 \\
\hline & 7 & 0.924 & 7.582 & 0.947 & 0.823 & 1.088 & 0.600 \\
\hline \multirow{3}{*}{ SHESEM } & 3 & $1 . \overline{228}$ & 22.813 & 1.133 & 0.895 & 1.231 & 0.787 \\
\hline & 5 & 0.936 & 6.393 & 1.200 & 0.739 & 1.065 & 0.720 \\
\hline & 7 & 0.936 & 6.385 & 1.211 & 0.877 & 1.070 & 0.720 \\
\hline \multirow{3}{*}{ SHESEE } & 3 & 1.044 & 4.356 & 1.200 & 0.733 & $1.23 \overline{1}$ & 0.620 \\
\hline & 5 & 0.934 & 6.575 & 1.150 & 0.875 & 1.065 & 0.691 \\
\hline & 7 & 0.954 & 4.629 & 1.211 & 0.742 & 1.053 & 0.677 \\
\hline
\end{tabular}




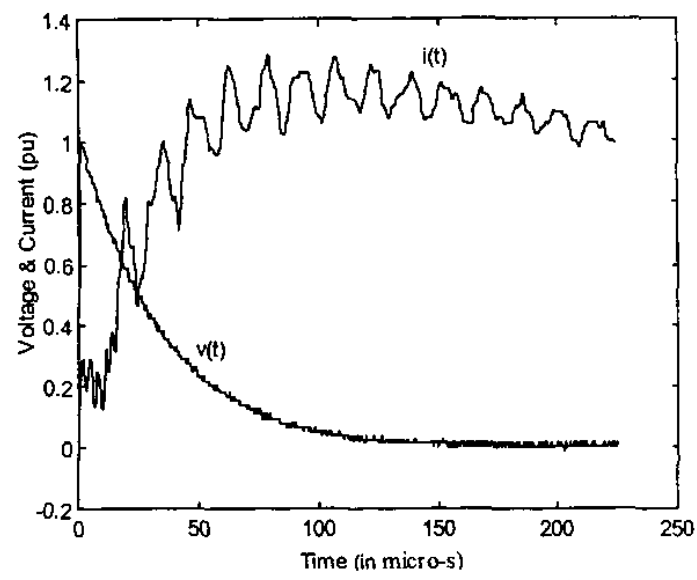

Figure 4. Typical voltage and current waveforms.

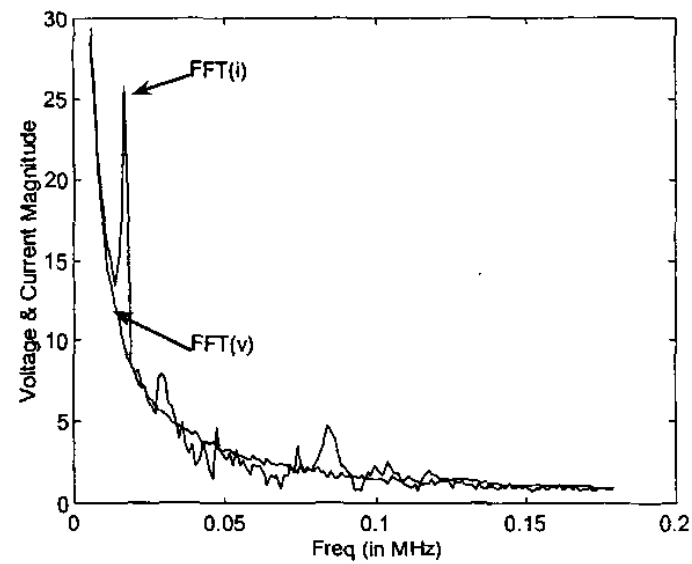

Figure 5. Frequency spectra of voltage and current waves shown in Figure 4.

TF curve. On the other hand, in the case of series fault at the mid-winding section, it is found that the successive resonant peaks of the BIL TF curve come closer to their calibrating TF counterparts at higher frequencies. The

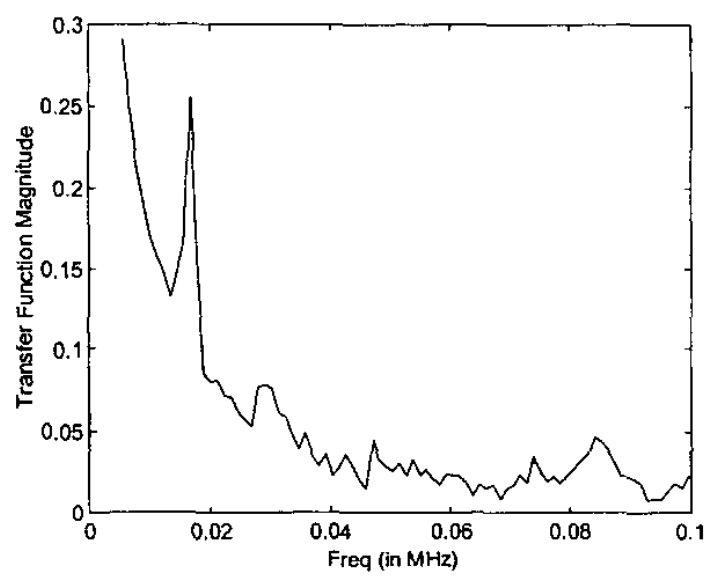

Figure 6. Typical Transfer Function of the winding of Figure 5. second pole magnitudes are however much attenuated in the BIL TF curve. The area under the BIL TF curve is found to be closer to the area under the calibrating $\mathrm{TF}$ curve.

In the case of series fault at the ground-end section, the first two resonant poles of the BIL TF curve deviates very little in frequency from their calibrating TF curve counterparts but the pole magnitude of the BIL TF curve deviate from those of the calibrating TF curve.

\subsubsection{SHUNT FAULTS}

Shunt fault at the line-end section results in a major reduction in the magnitude of the BIL TF curve at all frequencies. The various resonance peaks are heavily attenuated, and are almost absent in the BIL TF curve. As the position of the shunt fault shifts towards the midwinding section, the magnitude of the TF curve increases resulting in an increase in the second pole height of the TF curve as compared to the previous case of line-end shunt fault. However, in the TF curve at BIL, the first pole is found to be largely attenuated and in some cases it is totally absent.

Shunt fault at the ground end section results in a reduction of the BIL TF curve magnitude after $25 \mathrm{kHz}$. At BIL, the first resonance peak is close in magnitude to its calibrating counterpart but the second pole magnitude is much attenuated.

\subsubsection{SIMULTANEOUS FAULTS}

The method of diagnosis of simultaneous occurrence of shunt and series faults with the help of frequency domain analysis is described below.

SHLSE: As in the case of time domain analysis, it has been found in the frequency domain analysis also that the TF curve for SHLSE at BIL is very similar in nature to that of SHL at BIL and it has been observed that it is very difficult to differentiate between SHLSE and SHL TF curves.

SHMSEL: Shunt fault at the mid-winding section along with simultaneous series fault at the line-end section cause huge attenuation of the first pole height in the BIL TF curve but the second pole height is more in the BIL TF curve than the calibrating TF curve. The second pole frequencies, however, are very close in the two TF curves corresponding to BIL and calibrating level.

SHMSEM: In the case of simultaneous shunt and series faults at mid-winding section the first pole is very nearly absent and the second pole is greatly attenuated in the BIL TF curve as compared to the calibrating TF curve.

SHMSEE: If the location of the series fault shifts towards the ground end with the shunt fault occurring at the mid-winding section, there is an increase in the second pole height of the BIL TF curve as compared to that 
for SHMSEM, but the second pole is found to occur at a much lower frequency in the BIL TF curve as compared to the calibrating TF curve.

SHESEL: Simultaneous occurrence of shunt fault at ground-end and series fault at line end is characterized by large attenuation of the first two pole heights and shifting of the second pole frequency to a higher value at BIL with respect to that in the TF curve at calibrating level. The area under the BIL TF curve is less than that under the calibrating TF curve.

SHESEM: With the shunt fault occurring at the earth end, if the series fault occurs at the mid-winding section, then it is found that the area under the BIL TF curve deviates from the area under the calibrating TF curve. The second pole magnitude in the BIL TF curve is less attenuated than the case for SHESEL. Along with this, the first two resonance frequencies shift towards a comparatively higher value at BIL as compared to the TF curve at calibrating level.

SHESEE: A simultaneous shunt and series fault at the ground-end section is characterized by a large attenuation of the pole heights at BIL, however the attenuation of the second pole is less than that for SHESEL and more than that for SHESEM. It is also found that the first two resonance frequencies are higher in the BIL TF curve than those in the calibrating TF curve.

Logically, it follows that any fault, which may be diag. nosed unambiguously, using time domain analysis, can be reconfirmed with the help of frequency domain analysis.

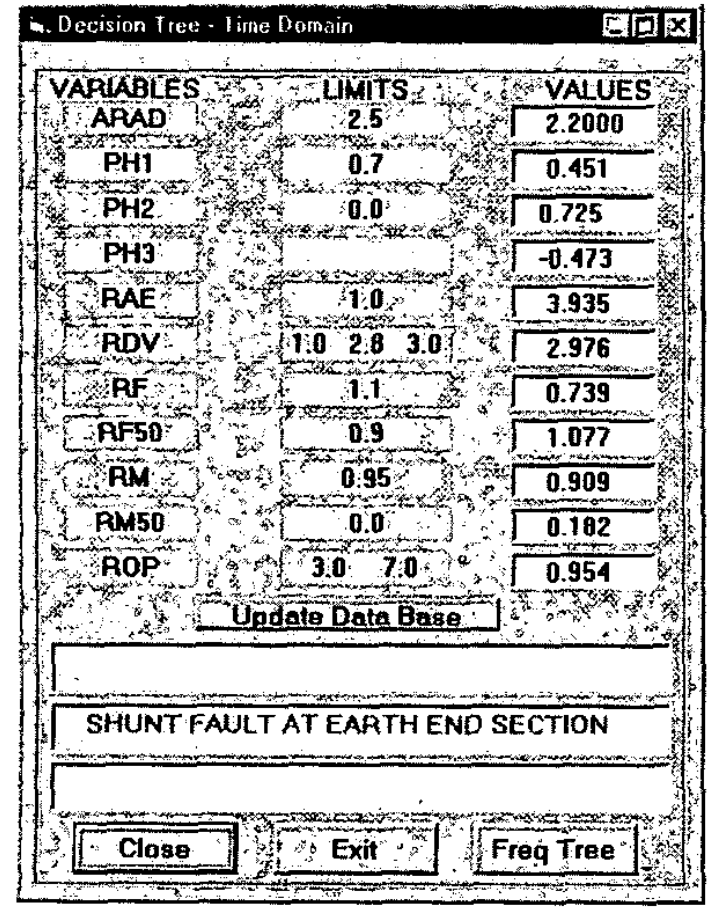

Figure 7. Explanation module through TAFEDIFT UI.
In this context, however, it should also be kept in mind that frequency domain analysis, notwithstanding its advantages, has certain disadvantages, too, which are due to the effects of noise, errors due to analog to digital converters $(\mathrm{ADC})$, windowing, sampling etc. Considering all the facts, it may therefore be stated that it is better not to depend solely upon any one method of analysis to arrive at a decision for impulse fault diagnosis. Consequently, the expert system TAFEDIFT employs both time and frequency domain analyses to supplement each other in the process of decision making, which leaves very little room for confusion in impulse fault diagnosis in high voltage transformers.

The algorithm of fault-detection, identification and its location determination by frequency-domain analysis, has also been presented in the integrated flow-chart of Figure 3 , the variables used therein being discussed in Appendix I.

\subsection{INFERENCE ENGINE}

An inference engine capable of performing logical reasoning on the rules in the rule-base and statistical methods has been designed for fault diagnosis. The inference engine proceeds as follows

1. Uses the rules in the time-domain rule-base to identify and locate the fault based on the data in the data-base.

2. Uses the rules in the frequency-domain rule-base to identify and locate the fault based on the data in the data-base.

3. Flashes the fault indication obtained from steps (i) and (ii).

4. Provide the user with the logic behind the fault diagnosis through the explanation module.

\subsection{EXPLANATION MODULE}

Whenever the expert system detects a fault, it immediately flashes a fault indication on the screen to the user. Subsequently if the user wants to find out the logic behind the inference drawn, he may do so through the explanation module. Through the UI, the expert system informs the user about the decision-making path used by its inference engine as shown in Figure 7.

\subsection{UPDATING MODULE}

Once a new set of raw data is acquired through experimentation or simulation, it goes through signal-conditioning filters incorporated using MATLAB, before being added to the data-base. Due to the modular construction of the software, the rule-base of the expert system can be updated and also expanded. 


\section{IMPLEMENTATION OF TAFEDIFT}

The expert system has been implemented on a PC at the HV Laboratory of Jadavpur University. To demonstrate the effectiveness of the designed expert system, impulse fault diagnosis has been performed on an analog model of a $33 / 11 \mathrm{kV}, 3 \mathrm{MVA}$, and EMTP simulated models of a $33 / 11 \mathrm{kV}, 5 \mathrm{MVA}$ and a $20 / 6 \mathrm{kV}, 7$ MVA transformers. In all three cases, it has been considered that the $\mathrm{HV}$ winding was subjected to impulse test voltages. The parameters of the transformer models are presented in Appendix II.

In the present study, voltage sensitive disc-to-disc short circuit faults involving $1 \%$ of the total winding length at a time has been simulated to represent series faults and disc-to-ground faults have been simulated to represent shunt faults. Faults have been simulated in discs over the entire length of the winding to properly represent the three winding sections, namely the line-end, mid-winding and ground-end sections, each section comprising of $33.3 \%$ of the entire length of the winding. Performance of the 3 MVA transformer is studied with its whole winding being subjected to impulses from a Recurrent Surge Generator (RSG). The winding response, captured by the tank-current method, is acquired by the PC via RS-232 interface circuit through a Tektronix TDS $320,100 \mathrm{MHz}, 500 \mathrm{MS} / \mathrm{s}$ digital storage oscilloscope. The block diagram of the entire experimental arrangement is presented in Figure 8, and the test set-up is shown in Figure 9. For the 5 MVA and 7 MVA transformers, all the different types of faults which were studied experimentally for the analysis of the 3 MVA transformer were simulated with EMTP models. EMTP model was also made for the 3 MVA transformer and the modeled values were found to be in good agreement with the experimental results. The results of fault diagnosis obtained for all the three transformers were used to calculate the fault detection parameters as shown in Appendix I. Tables 2 and 3 show calculated values of the fault detection parameters for different types of faults for all the three transformers employing the time-domain and frequency-domain analyses, respectively.

Figures 10 to 15 show typical displays of waveforms for the different transformers in the expert system UI. The

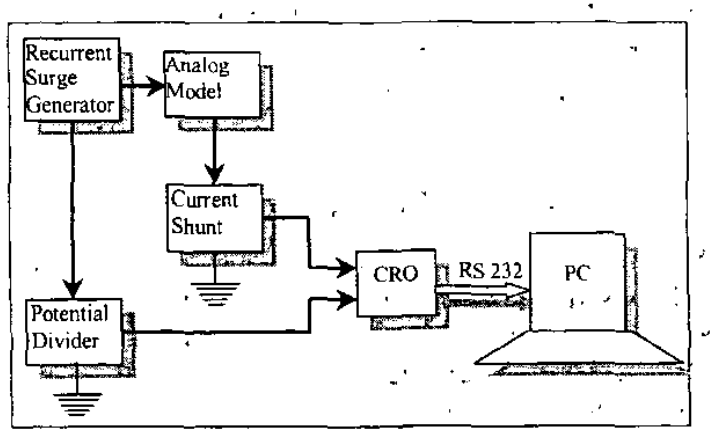

Figure 8. Block diagram of the experimental arrangement.

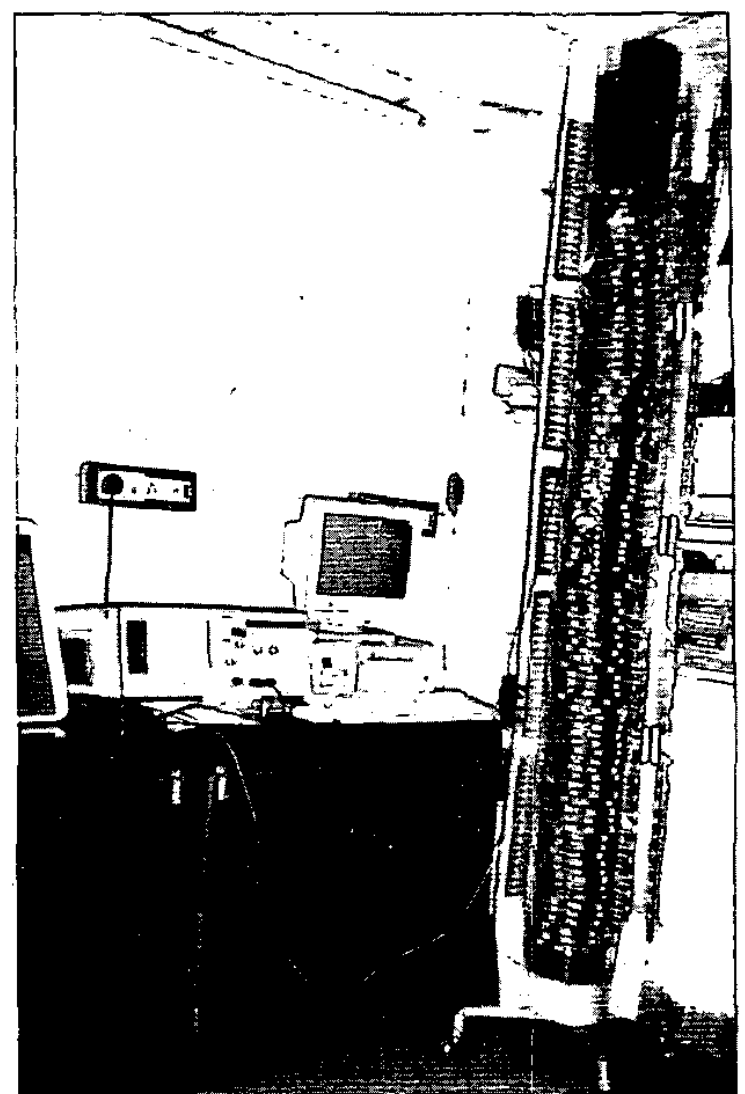

Figure 9. Test set-up with analog model of transformer winding.

inference engine of the designed expert system follows the rule-base described earlier to operate on the values of these parameters for fault diagnosis. The decision-tree followed by the inference engine of the expert system for fault diagnosis are displayed in each case in the explanation module window, a typical one of which is shown in Figure 7.

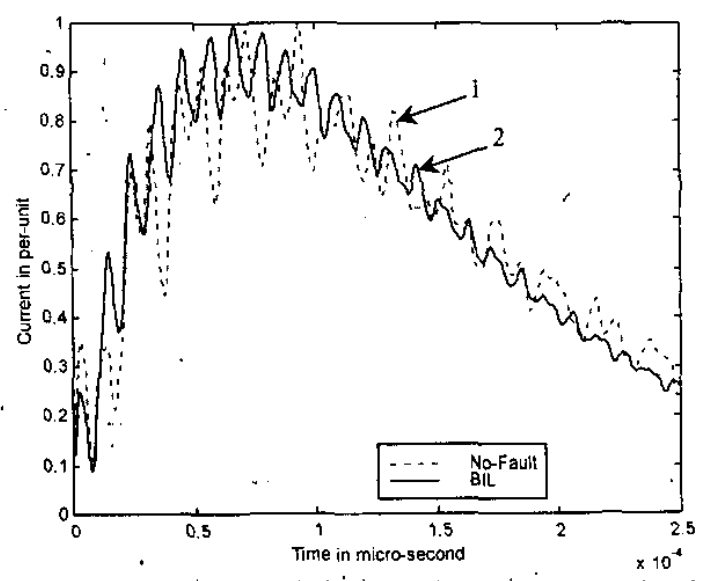

Figure 10. Time-Domain analysis of simultaneous shunt and series fault at mid-winding section for 3MVA transformer. 1, No fault; 2, BIL. 


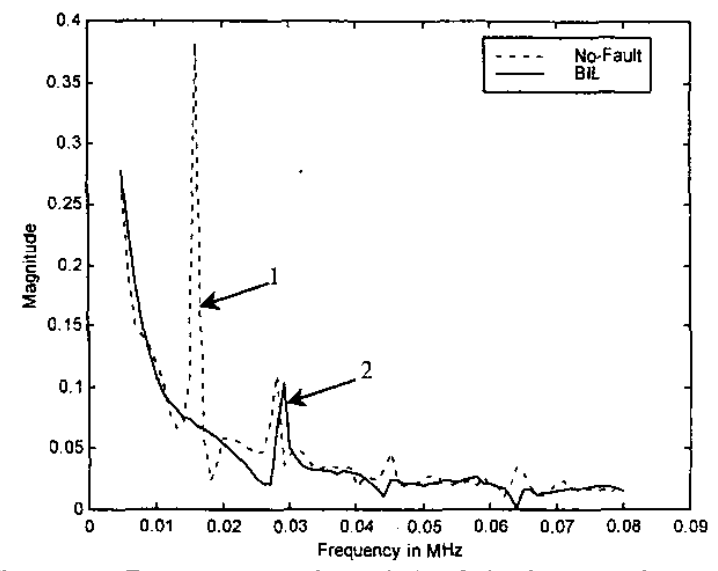

Figure 11. Frequency-Domain analysis of simultaneous shunt and series fault at mid-winding section for 3 MVA transformer. 1, No fault; 2, BIL.

\section{CONCLUSIONS}

T $\mathrm{N}$ this paper, an expert system has been developed $\boldsymbol{I}_{\text {to serve as an operational aid for reliable detection and }}$ localization of insulation failures during impulse tests in the range of power transformers commonly used up to 33 $\mathrm{kV}$. The embedded rule-base of the expert system consists of time-domain and frequency-domain (Transfer Function) modules for fault diagnosis. These modules form the basis for the re-confirmative deductive reasoning performed by the inference engine to identify and locate the insulation failure. The incorporation of both the time and frequency domain analyses ensures high reliability in the fault diagnosis process. The effectiveness of the Expert System has been demonstrated by impulse fault diagnosis on EMTP simulated models of a $33 / 11 \mathrm{kV}, 5 \mathrm{MVA}$ and a $20 / 6 \mathrm{kV}, 7$ MVA transformers. The testing of the developed expert system has also been done on an analog model of a $33 / 11 \mathrm{kV}, 3$ MVA transformer and tests on real trans-

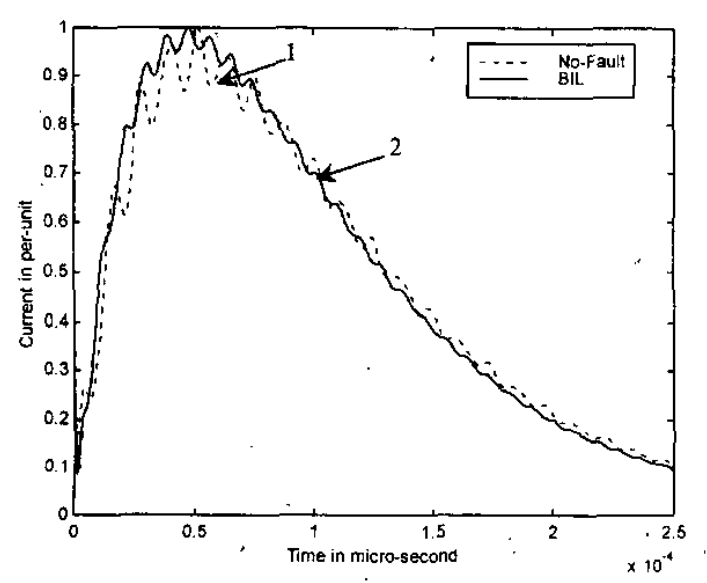

Figure 12. Time-Domain analysis of shunt fault at mid-winding sec= tion for 5 MVA transformer. 1, No fault; 2, BIL.

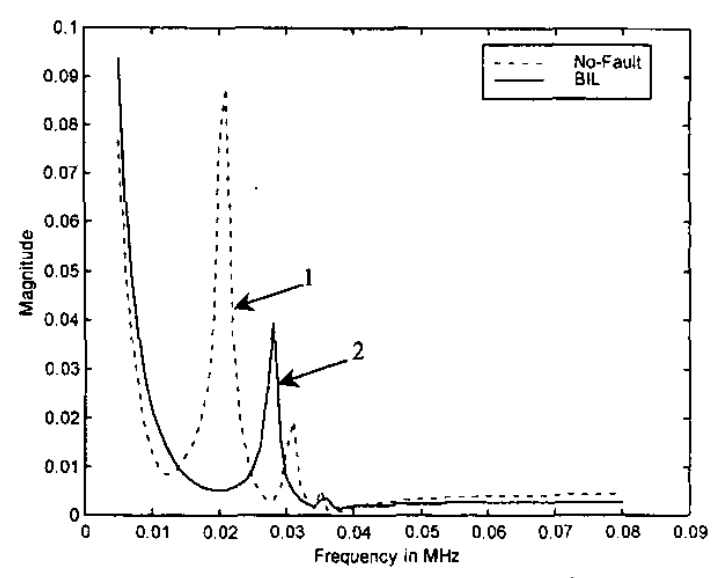

Figure 13. Frequency-Domain analysis of shunt fault at mid-winding section for 5 MVA transformer. 1, No fault; 2 , BIL.

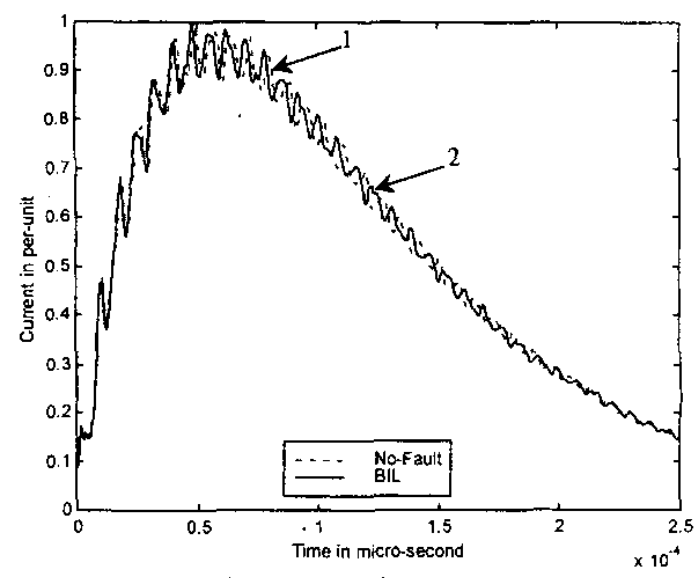

Figure 14. Time-Domain analysis of series fault at mid-winding section for 7 MVA transformer. 1, No fault; 2, BIL.

formers of different ratings may be of help to prove further the usefulness of the method.

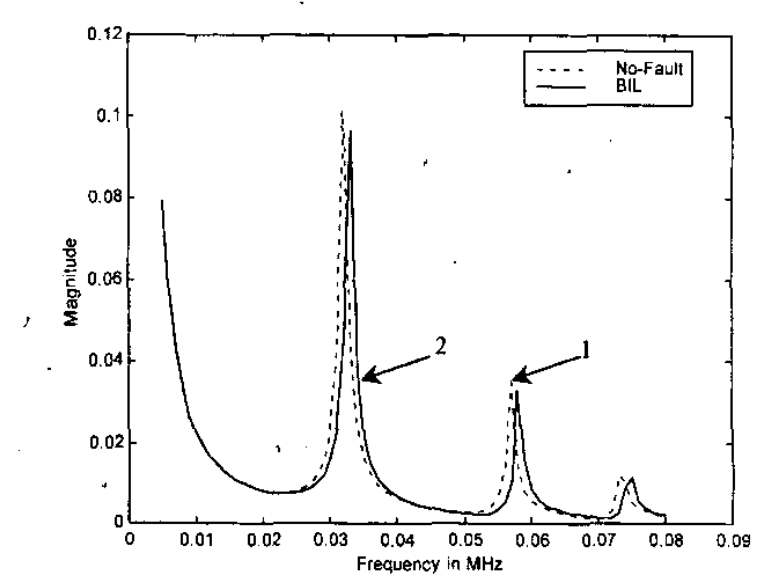

Figure 15. Frequency domain analysis of series fault at mid-winding section for 7 MVA transformer. 1, No fault; 2, BlL. 


\section{APPENDIX I}

\subsection{VARIABLES USED IN THE FAULT-DETECTION ALGORITHMS}

\subsubsection{TIME DOMAIN}

ARAD: Absolute percentage deviation in the area under the BIL current wave from the calibrating current wave.

PH1: Sum of deviations in the times of occurrence of the respective peaks in the two current waves under comparison within $50 \mu \mathrm{s}$.

PH2: Sum of deviations in the times of occurrence of the respective peaks in the two current waves under comparison between 50 to $150 \mu \mathrm{s}$.

PH3: Sum of deviations in the times of occurrence of the respective peaks in the two current waves under comparison beyond $150 \mu \mathrm{s}$.

In the above three cases the parameters $\mathrm{PH} 1, \mathrm{PH} 2$ and $\mathrm{PH} 3$ have negative values for leading BIL waves and positive values for lagging BIL waves.

RAE: Ratio of average absolute errors of the two current waves under comparison, where Average Absolute Error is defined as

$$
A A E=\frac{1}{N} \sum|(C 1-C 2)|
$$

where $C 1$ and $C 2$ are ordinates for same abscissa for the two current or voltage waveforms under comparison and $N$ is the number of data points in a wave.

RDV: Ratio of Distance Vectors calculated for calibrating level and BIL current and also impressed voltage waves. The Distance Vector is defined as

$$
D V=\frac{1}{N} \sqrt{\Sigma}(C 1-C 2)^{2}
$$

RF: Ratio of frequency of oscillation of the two current waves up to $50 \mu \mathrm{s}$.

RF50: Ratio of frequency of oscillation of the two current waves after $50 \mu \mathrm{s}$.

RM: Ratio of average peak-to-peak oscillation magnitude of the two current waves under comparison up to $50 \mu \mathrm{s}$.

RM50: Ratio of average peak-to-peak oscillation magnitude of the two current waves under comparison after $50 \mu \mathrm{s}$.

ROP: Ratio of the number of peaks of oscillation in the two current waves under comparison.

\subsubsection{FREQUENCY DOMAIN}

ARA: Ratio of areas under the curve for the two TF curves.

ADV: Percentage deviation in the areas under the curve between the two TF curves.

FF: Ratio of first resonance frequencies of the two TF curves.

FM: Ratio of magnitudes of first resonant peak of the two TF curves.

SF: Ratio of second resonance frequencies of the two TF curves.

SM: Ratio of magnitudes of second resonant peak of the two TF curves.

The magnitudes of all the above-mentioned variables are calculated considering the waveform at BIL and that at the calibrating level.

\section{APPENDIX II}

\subsection{TRANSFORMER MODEL PARAMETERS}

\begin{tabular}{llll}
\hline & 3 MVA & 5 MVA & 7 MVA \\
\hline V & $33 / 11$ & $33 / 11$ & $20 / 6$ \\
OD & 524 & 609 & 702 \\
ID & 424 & 503 & 579 \\
W & 50 & 53 & 61.5 \\
H & 6.6 & 17.8 & 10.0 \\
$\mathrm{~N}$ & 90 & 37 & 34 \\
$\mathrm{~T}$ & 19 & 32 & 16 \\
\hline
\end{tabular}

\subsection{SYMBOLS FOR 6.1}

\begin{tabular}{cll}
\hline Symbol & \multicolumn{1}{c}{ Variable } & Unit \\
\hline V & $\begin{array}{l}\text { Voltage Rating } \\
\text { Outer Diameter of HV } \\
\text { winding }\end{array}$ & $\mathrm{kV}$ \\
$\mathrm{mm}$ \\
$\mathrm{ID}$ & $\begin{array}{l}\text { Inner Diameter of HV } \\
\text { winding }\end{array}$ & $\mathrm{mm}$ \\
$\mathrm{W}$ & $\begin{array}{l}\text { Radial thickness of disc } \\
\text { Axial height of disc }\end{array}$ & $\mathrm{mm}$ \\
$\mathrm{H}$ & $\mathrm{mm}$ \\
$\mathrm{N}$ & $\begin{array}{l}\text { Number of discs } \\
\text { Turns per disc }\end{array}$ & \\
\hline
\end{tabular}

\section{ACKNOWLEDGMENT}

$\therefore$ The authors would like to express their thanks to "All India Council for Technical Education' for financially sup*porting this work through project grant (8017/RDII/ ELE/203/98) to Dr. S. Chakravorti during 1998-2000. 


\section{REFERENCES}

[1] "Power Transformer - Insulation Levels and Dielectric Tests", IEC Publication 76-3, 1980.

[2] "Guide to the Lightning and Switching Impulse Testing of Power Transformers and Reactors", IEC Standard, Publication 722, 1982.

[3] F. Beldi, "The Impulse Testing of Transformers", The Brown Boveri Review, Vol. 37, pp. 179-193, 1950.

[4] J.H. Hagenguth and J.R. Meador, "Impulse Testing of Power Transformers”, AIEE Trans., Vol. 71, pp. 697-704, 1952.

[5] L.C. Aicher, "Experience With Transformer Impulse Failure Detection Methods", AIEE Trans., Vol. 67, pp. 1621-1631, 1948.

[6] E. Stenkvist, "Study of Fault Detection and Failure Location During Surge Testing of Transformers", CIGRE, Supplement to paper No. 12-125, 1952.

[7] G.B. Harper, "Detection and Diagnosis of Deterioration and Faults in Power Transformers", CIGRE, paper 12.01, pp. 19-25, 1967.

[8] C.K. Roy and J.R. Biswas, "Studies on Impulse Behavior of a Transformer Winding With Simulated Faults by Analogue Modeling", IEE Proceedings-C, Vol. 141, pp. 401-412, 1994.

[9] R. Malewski and B. Poulin, "Impulse Testing of Power Transformers Using the Transfer Function Method", IEEE Trans. Power Delivery, Vol. 3, pp. 476-490, 1988.

[10] P.T.M. Vaessen and E. Hanique, "A New Frequency Response Analysis Method for Power Transformers", IEEE Trans. Power Delivery, Vol. 7, pp. 384-390, 1992.

[11] D.Tschudi, "Location of Power Transformer Faults During the Impulse Test", Thesis No:1168 ( 1993 ) - Department of Electricity, Federal Institute of Technology, Lausanne, Switzerland.

[12] R.Vajana and K. Udayakumar, "A New Paradigm for Impulse Testing of Power Transformers", IEEE PES Winter Meeting, paper No. 15-01-08, Singapore, 2000.
[13] R.Vajana and K. Udayakumar, "Fault Location in Power Transformers During Impulse Tests", IEEE PES Winter Meeting, paper no. 15-01-06, Singapore, 2000.

[14] J. Bak-Jensen, B. Bak-Jensen and S.D. Mikkelsen, "Detection of Faults and Aging Phenomena in Transformers by Transfer Functions", IEEE Trans. Power Delivery, Vol. 10, pp. 308-314, 1995.

[15] S. K. Pandey and L. Satish, "Multi Resolution Signal Decomposition: A New Tool for Fault Detection in Power Transformers During Impulse Tests", IEEE Trans. Power Delivery, Vol. 13, pp. 1194-1200, 1998.

[16] B. F. Wollenberg and T. Sakaguchi, "Artficial Intelligence in Power System Operation", IEEE Proceedings, Vol. 75, pp. 1678-1685, 1987.

[17] Y. Hsu, F.C. Lu, Y. Chien, J.P.Liu, J.T. Lin, H.S. Yu and R.T Kuo, "An Expert System For Locating Distribution System faults", IEEE Trans. Power Delivery, Vol. 6, pp. 366-371, 1991.

[18] Z. Wang, Y. Liu and P.J. Griffin, "A Combined ANN and Expert System Tool For Transformer Fault Diagnosis", IEEE Trans. Power Delivery, Vol. 13, pp. 1224-1229, 1998.

[19] P. Purkait and S. Chakravorti, "An Expert System for Fault Diagnosis in Transformers During Impulse Tests", IEEE PES Winter Meeting, paper No. 15-01-03, Singapore, 2000.

[20] M.A. Sanz-Bobi, A. Garica-Cerrada, J. Rolan and A. Luengo, "TRAFES : An Expert System for Diagnosis of Power Transformcrs", Proceedings of CIGRE symposium, Berlin, paper No. 110-20, pp. 1-6, 1993.

[21] C.E. Lin, J.M. Ling and C.L. Huang, "An Expert System for Transformer Fault Diagnosis using Dissolved Gas Analysis", IEEE Trans. Power Delivery, Vol. 8, pp. 231-238, Jan. 1993.

Manuscript received on 31 July 2000, in final form 18 February 2002. 